UCRL-ID-127786

\title{
The Rubble Rescue Radar (RRR): A Low Power Hand-Held Microwave Device for the Detection of Trapped Human Personnel
}

\author{
W. S. Haddad
}

April 10, 1997

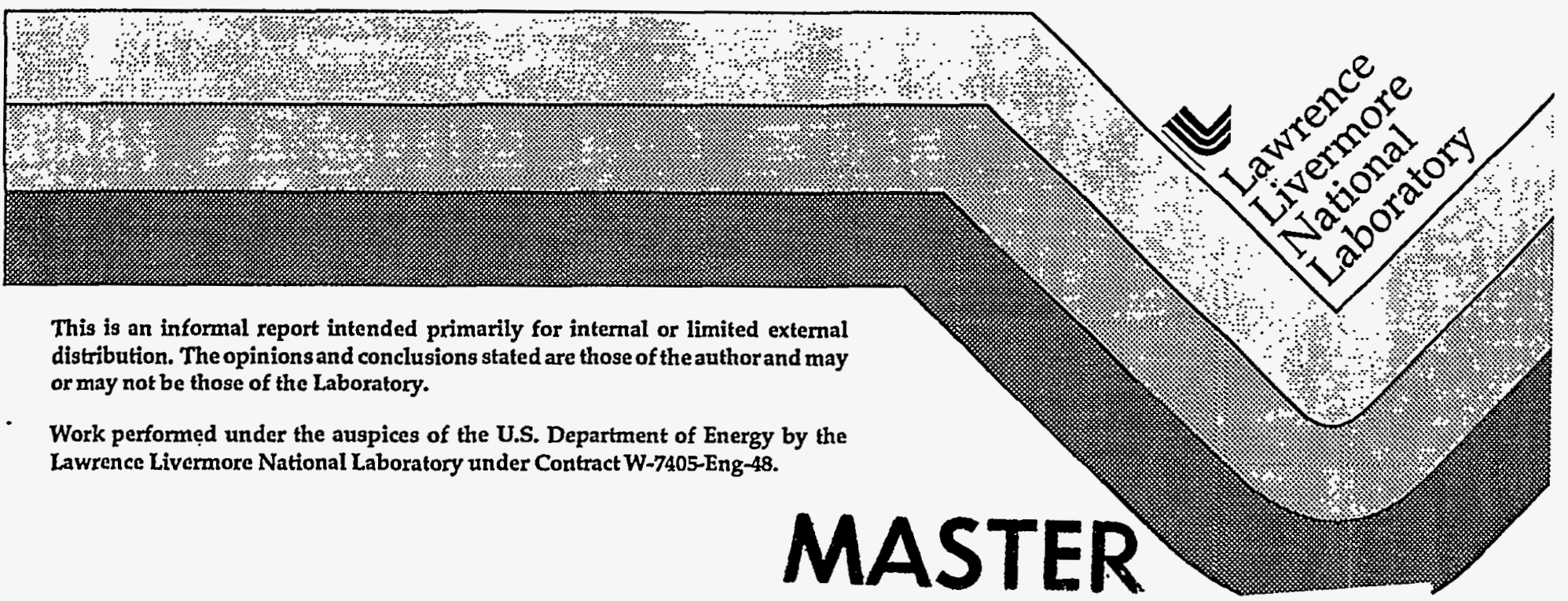

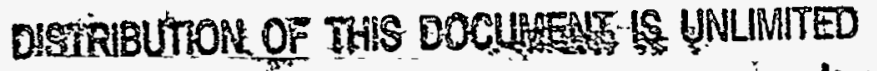




\section{DISCLAIMER}

This document was prepared as an account of work sponsored by an agency of the United States Government. Neither the United States Government nor the University of California nor any of their employees, makes any warranty, express or implied, or assumes any legal liability or responsibility for the accuracy, completeness, or usefulness of any information, apparatus, product, or process disclosed, or represents that its use would not infringe privately owned rights. Reference herein to any specific commercial product, process, or service by trade name, trademark, manufacturer, or otherwise, does not necessarily constitute or imply its endorsement, recommendation, or favoring by the United States Government or the University of California. The views and opinions of authors expressed herein do not necessarily state or reflect those of the United States Government or the University of California, and shall not be used for advertising or product endorsement purposes.

This report has been reproduced directly from the best available copy.

Available to DOE and DOE contractors from the Office of Scientific and Technical Information

P.O. Box 62, Oak Ridge, TN 37831

Prices available from (615) 576-8401, FIS 626-8401

Avallable to the public from the

National Technical Information Service

U.S. Department of Commerce

5285 Port Royal Rd.,

Springfield, VA 22161 


\section{DISCLAIMER}

Portions of this document may be illegible electronic image products. Images are produced from the best available original document. 


\title{
The Rubble Rescue Radar (RRR): A Low Power Hand-Held Microwave Device for the Detection of Trapped Human Personnel
}

\author{
W. S. Haddad
}

$4 / 10 / 97$

Lawrence Livermore National Laboratory

P.O. Box 808, L-399 Livermore CA 94551

\begin{abstract}
Each year, innocent human lives are lost in collapsed structures as a result of both natural and man-made disasters. We have developed a prototype device, called the Rubble Rescue Radar (RRR) as a aid to workers trying to locate trapped victims in urban search and rescue operations. The RRR is a motion sensor incorporating Micropower Impulse Radar (MIR), and is capable of detecting human breathing motions through reinforced concrete. It is lightweight, and designed to be handled by a single operator for localized searches in areas where trapped victims are expected. Tests of the first prototype device were conducted on site at LLNL using a mock rubble pile consisting of a reinforced concrete pipe with two concrete floor slabs placed against one side, and random concrete and asphalt debris piled against the other. This arrangement provides sáfe and easy access for instruments and/or human subjects. Breathing signals of a human subject were recorded with the RRR through one floor slab plus the wall of the pipe, two slabs plus the wall of the pipe, and the random rubble plus the wall of the pipe. Breathing and heart beat signals were also recorded of a seated human subject at a distance of 1 meter with no obstructions. Results and photographs of the experimental work are presented, and a design concept for the next generation device is described.
\end{abstract}

Keywords: urban search and rescue, breathing motion, Micropower Impulse Radar (MIR), rubble.

\section{Introduction}

Bvery year, around the world, innocent human victims are trapped in rubble or collapsed structures as a result of both natural and manmade catastrophes. In all too many cases, these victims die as a direct or indirect result of their circumstance. Death can result from injuries sustained during the collapse, lack of air, water or food in cases of prolonged entrapment. Trapped victims are sometimes killed accidentally by shifting or falling rubble during the rescue operations, or by heavy equipment during removal of parts of the collapsed structure when workers are unaware of the presence of a live victim in their path.

In all cases, the sooner a living victim is located, the greater his or her chances are for survival. While speed is critical in locating victims, so is the reliability of the method of location. While rescue workers labor fruitlessly digging in the wrong place or reaching someone who is already dead, others' chances for survival dwindle. There is no method for finding trapped victims which is fast, safe, and highly reliable. At present, the single most effective urban search and rescue (USAR) method for finding trapped victims in the midst of rubble is the use of trained sniffer dogs. The highly-trained dogs and their expert trainers are in short supply. The costs of training the dogs and having them and their trainers available for emergency rescue operations are quite high, and are generally not covered by government funding; the job is therefore typically left up to dedicated volunteers.

What is needed is a new device or technique for the detection of trapped victims that is low cost, light weight and portable, and capable of increasing the chances of finding a living victim early on in the USAR operations. Motivated by humanitarian concerns and the availability of microwave and signal processing expertise at Lawrence Livermore National Laboratory (LLNL), we undertook to 

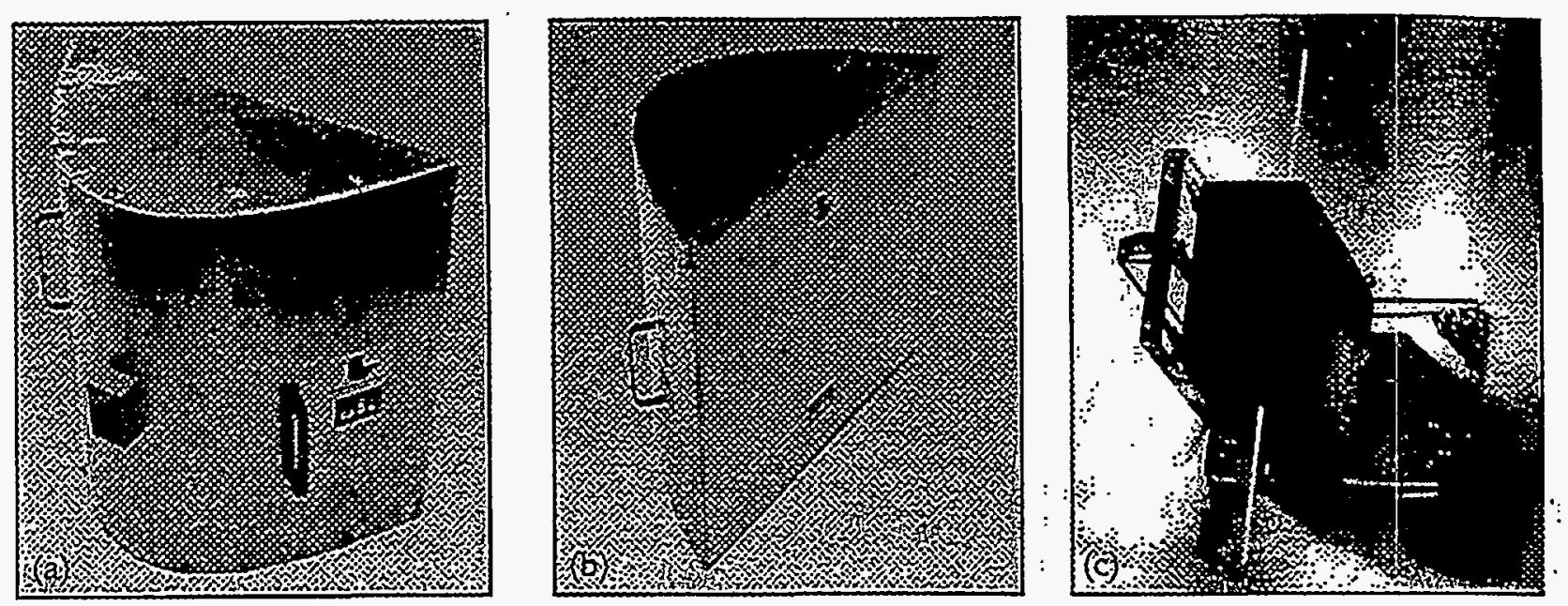

Figure 1. Photographs of the RRR. (a) and (b) two views of the RRR, (c) close up of the MIR transmitter/receiver $(T / R)$. The $T / R$ is positioned with its antenna along the line focus of the parabolic reflector.

develop such a device in collaboration with the Army Corps of Engineers' Earthquake Preparedness Center of Expertise in San Francisco, California. The first prototype device is based on Micropower Impulse Radar (MIR), a new technology developed at LLNL over the past three years [1].

MIR devices are small, lightweight, inexpensive to manufacture and have very low power consumption, allowing it to operate continuously for extended periods on a small battery. They can produce microwave bursts or low power microwave pulses as short as 150 picoseconds in duration. MIR devices contain a transmitter and a receiver circuit, and work by first transmitting a short pulse, then turning on the receiver circuit momentarily after a time delay, $T$. The receiver detects the return pulse reflected from any object or interface a distance $\mathrm{c} / 2 \mathrm{~T}$ away, where $\mathrm{c}$ is the speed of light in the surrounding medium. In this way, MIR devices can be "range-gated" to eliminate unwanted returns from objects which are either too close or too far. Typically pulses are transmitted with a pulse repetition frequency (PRF) of about $2 \mathrm{MHz}$. Many pulses can be integrated to increase signal-to-noise. If the time delay $\mathrm{T}$ is incremented by $\delta \mathrm{T}$ at a rate much smaller than the PRF, a time history of the return pulses can be generated. Systems have been produced which have a nominal pulse width of about 150 picoseconds and a $\delta \mathrm{T}$ of approximately 7 picoseconds. The microwave pulses produced by MIR devices typically have a peak frequency of $2 \mathrm{GHz}$, with a bandwidth of 2 to $3 \mathrm{GHz}$. Penetration into the body has been demonstrated in a number of recent experiments [2], and signals from the beating of the heart and the motion of speech articulators have been recorded in human subjects [3,4]. The level of microwave radiation produced by these devices has been measured by LLNL safety officers and was found to be several orders of magnitude below the acceptable limit for human exposure.

\section{First Generation RRR \\ Specifications and Properties}

The first version of the RRR was designed with a specially modified MIR motion sensor as its detector system. Several views of the prototype $R R R$ is shown in Figure 1. Figure $1 c$ is a close up of the MIR "feed" located in the reflector dish. Table 1 contains a list of some of the specifications of the RRR protofype.

Table 1.

Prototype RRR Specifications

\begin{tabular}{ll}
\hline Reflector: & 1-dimensional parabola \\
material & aluminum \\
focal length & $7.5 \mathrm{~cm}$ \\
depth & $30 \mathrm{~cm}$ (apex to aperture) \\
aperture & $60 \mathrm{~cm}$ \\
Feed: & $M \mathrm{RR}$ motion sensor \\
center frequency & $1.3 \mathrm{Ghz}$ \\
power & one standard 9 V. cell \\
antenna & end-fed monopole \\
duration & $\sim 2 \mathrm{hr}$. per battery \\
Overall weight & $\sim 6 \mathrm{Kg}$. \\
\hline
\end{tabular}



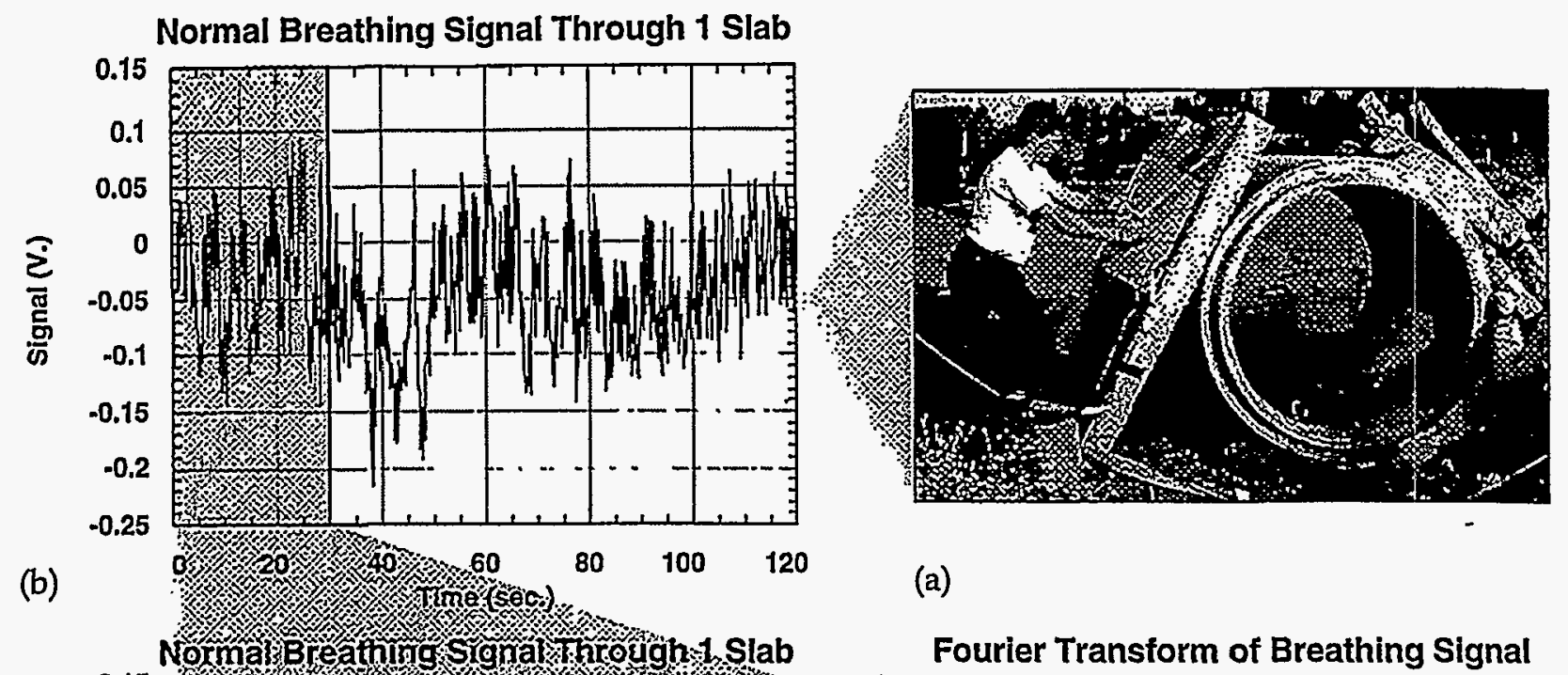

(a)

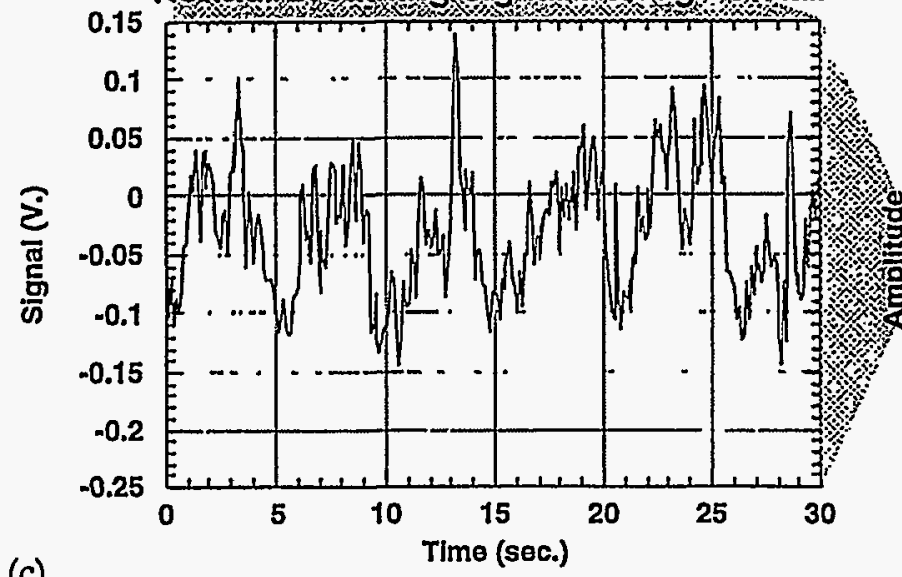

(c)

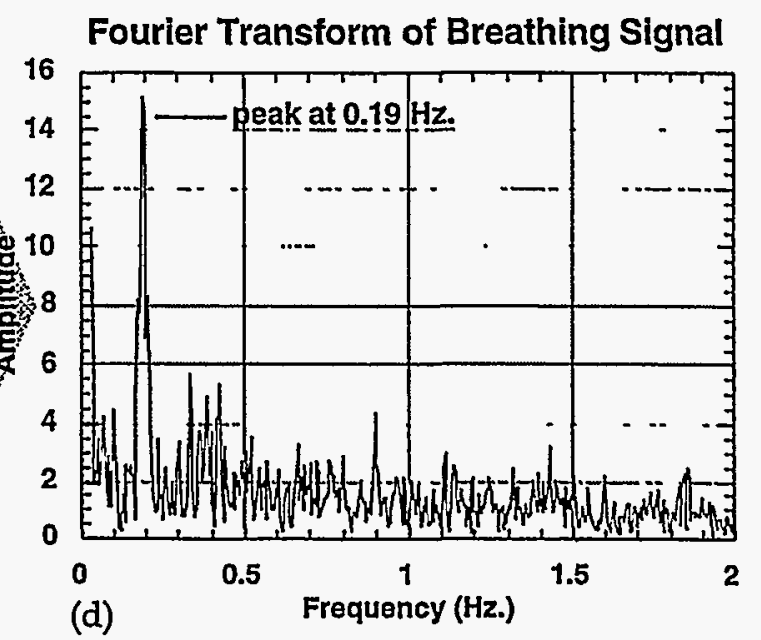

FIGURE 2. Breathing signals of a human subject recorded with the RRR through a single reinforced concrete floor slab and a concrete pipe. (a) a photograph showing breathing detection experiments at the rubble pile testbed located on site at LLNL. (b) the raw signal as recorded by the RRR. (c) expanded plot of the highlighted region in (b). The breathing motion is clearly visible as a large modulation in the signal with a period of about 5 seconds. (d) the Fourier transform of the raw signal showing a prominent peak at the breathing frequency.

The geometric profile of the reflector is parabolic in one dimension and flat in the other. This causes it to have a focal line running parallel to the flat axis. The reflector was designed to function as a collimator, and specified for maximum gain assuming a center frequency of the feed at $1 \mathrm{GHz}$. This means that the focal line is located $7.5 \mathrm{~cm}$ from the apex of the parabola, which corresponds to $1 / 4$ wavelength at $1 \mathrm{GHz}$. The feed is built with monopole antennas which have a radiation pattern similar to that of a dipole. Exploiting the dipole radiation pattern, the feed is positioned with the antennas lying along the focal line of the parabola.

\section{Experimental Tests with the RRR and Results}

In order to conduct tests of the RRR's performance under conditions similar to those for which it was designed, a mock rubble pile was constructed on site at LLNL. This rubble pile testbed was designed so that instruments and/or human subjects could safely and easily be placed behind both concrete floor slabs as well as randomly distributed concrete, asphalt and rebar debris. Photographs of the rubble pile testbed are included in Figures 2, 3 and 4. The 
(a)
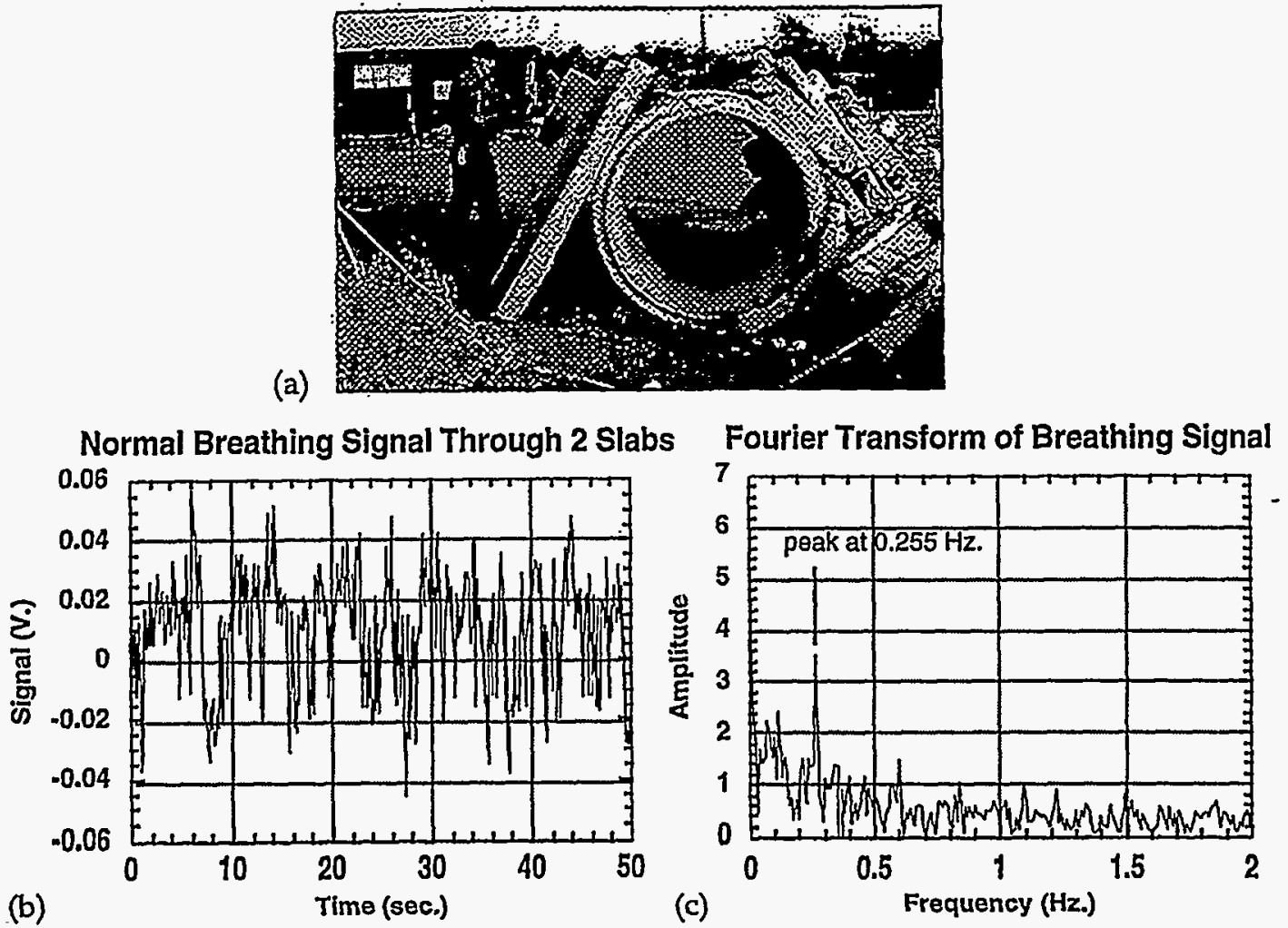

Figure 3. Breathing signals of a human subject recorded through two reinforced concrete slabs and the wall of a concrete pipe with the RRR. (a) a photograph of the experiments being conducted at the rubble pile testbed. (b) the raw signal recorded by the $R R R$. The breathing motion is less pronounced than in the single slab signal shown in Figure $2 b$, but still clearly present. (c) the Fourier transform of the raw signal showing a clear peak at the breathing frequency of $0.255 \mathrm{~Hz}$.

testbed consists of a concrete pipe with the rubble stacked up against the sides, leaving the open region of the pipe easily accessible. One side of the testbed has two floor slabs staggered such that measurements can be taken through either a single slab or both slabs. The floor slabs were designed and built to represent accurately those that are commonly found in high-rise buildings. They are 6" thick 3000 psi concrete with 1/2" diameter rebar on a square lattice with 6 " spacing. The concrete pipe is 4 feet in diameter, and is made in two 3foot-long sections butted together to create a 6 foot-long tunnel. The pipe wall is $5^{\prime \prime}$ thick $4000 \mathrm{psi}$ concrete with $1 / 2^{\prime \prime}$ rebar hoops spaced by $8^{\prime \prime}$. This meets the ASTM standards for D loading a 6 foot long tube with a force equivalent to the full weight of the two slabs.

Experiments involving human subjects were performed with the prototype RRR at the rubble pile testbed. The subjects were asked to enter the concrete tube, and to sit still. The RRR signals were then acquired during normal breathing, exaggerated breathing and occasionally with the subject moving his hand back and forth slightly. Similar experiments were performed with the RRR in different orientations and through various regions of the rubble pile testbed.

Figure 2 shows an example of the signals recorded through a single floor slab. The photograph of the experiment in Figure $2 a$ of the experiment clearly shows the rubble pile testbed, the placement of the radar and the location of the subject. The raw signal as recorded by the $R R R$ is shown in Figure $2 b$ and its Fourier transform (FT) is shown in Figure 2d. Figure 2c is a blowup of a section of the raw signal, clearly showing the modulation due to the breathing motion of the subject. A very clear peak corresponding to this motion can be seen in FT of the signal at the breathing frequency. The height of this peak compared to the background indicates the effectiveness of the RRR to detect the presence of the live subject.

A similar experiment was performed with the RRR looking through 2 slabs and the tube. This 


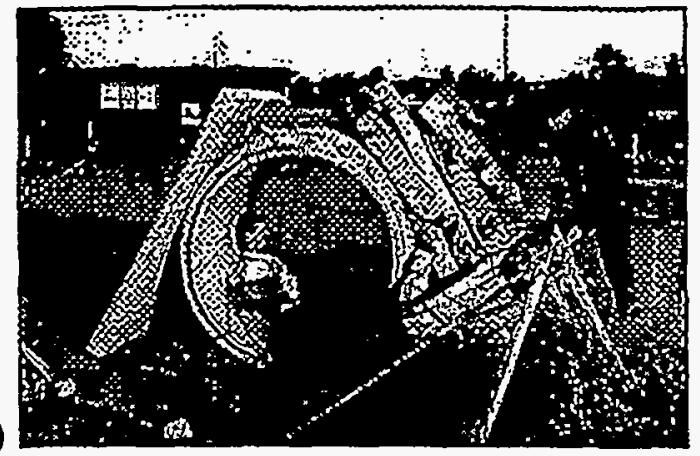

Exaggerated Breathing Signal Through Debris

(b)

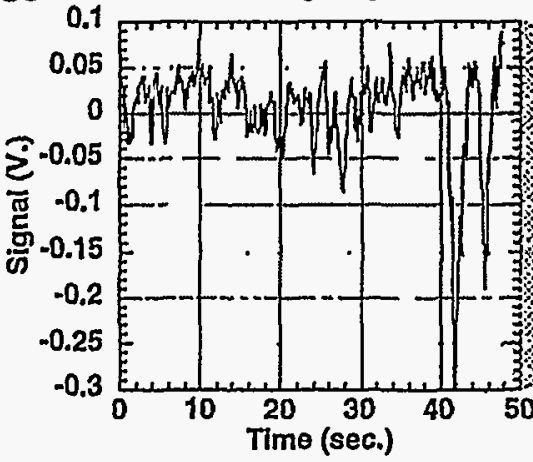

Fourier Transform of Breathing Signal

Normal Breathing Signal Through Debris

(d)

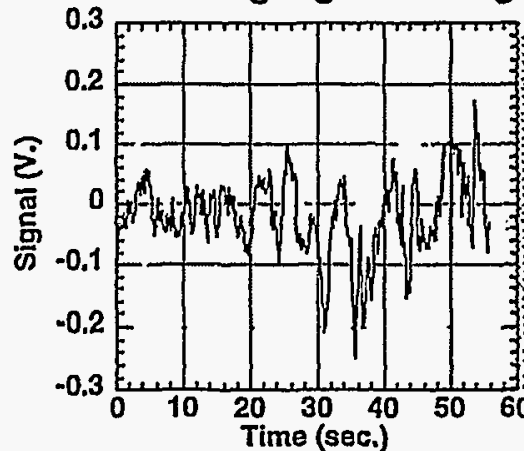

(c)

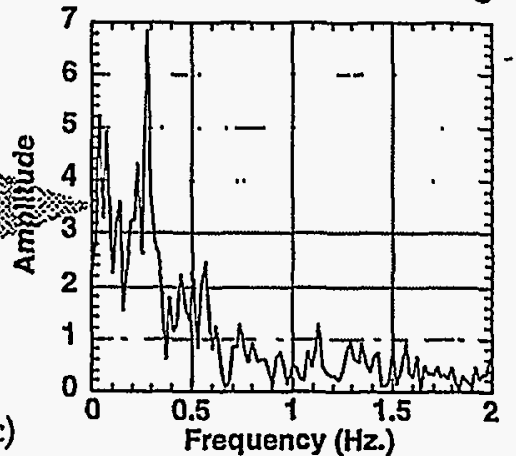

Fourier Transform of Breathing Signal

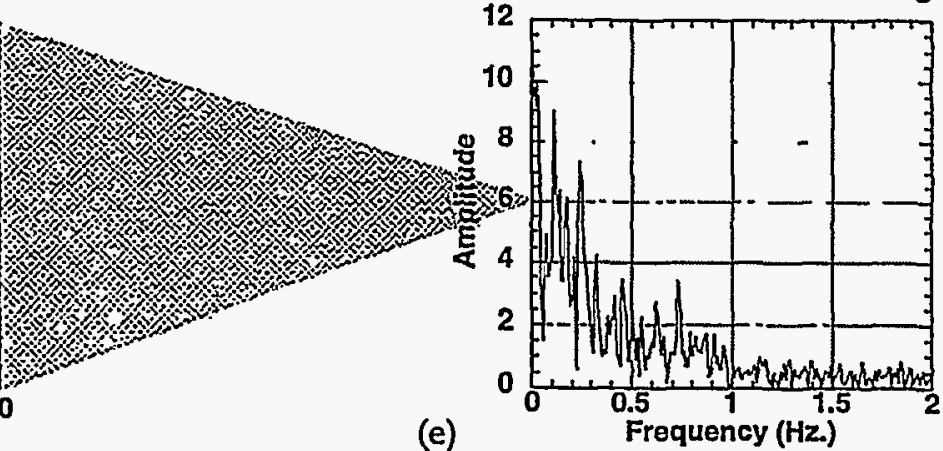

Eigure 4. Exaggerated and normal breathing signals of a human subject recorded with the RRR through randomly distributed rubble and a concrete pipe. (a) a photograph of the experiment. (b) and (c) the raw exaggerated breathing signal and its Fourier transform respectively. (d) and (e) the raw normal breathing signal and its Fourier transform respectively. A clear peak at the breathing frequency is seen for the exaggerated breathing motion in (c), however, the same peak is lost in the noise for normal breathing (e).

shown in Figure 3. Again Figure 3a is a photograph of the experiment, Figure $3 b$ and $3 c$ show the raw RRR signal and its FT respectively. Again a clear peak at the breathing frequency is seen in the FT, although with a significantly lower signal compared to the background.

Next, the RRR was tested through the random debris at the other side of the rubble pile testbed. This is shown in Figure 4. This was the most difficult test for the RRR. Figures $4 b$ and $c$ show the raw RRR signal and its FT respectively for exaggerated breathing of the subject, while Figures 4d and e show the RRR signal and its FT for normal breathing. It is apparent from these data that this scenario (depicted in Figure 4a) represents the threshold of the current RRR prototype's ability to detect a living subject. The breathing peak can be seen in the FT of the exaggerated breathing signal, but the same peak in the FT of the normal breathing is not clearly discernible from the noise.

As an additional test and further demonstration of the RRR's capabilities, we measured signals from a human subject through air only. The subject was seated in a chair and placed 

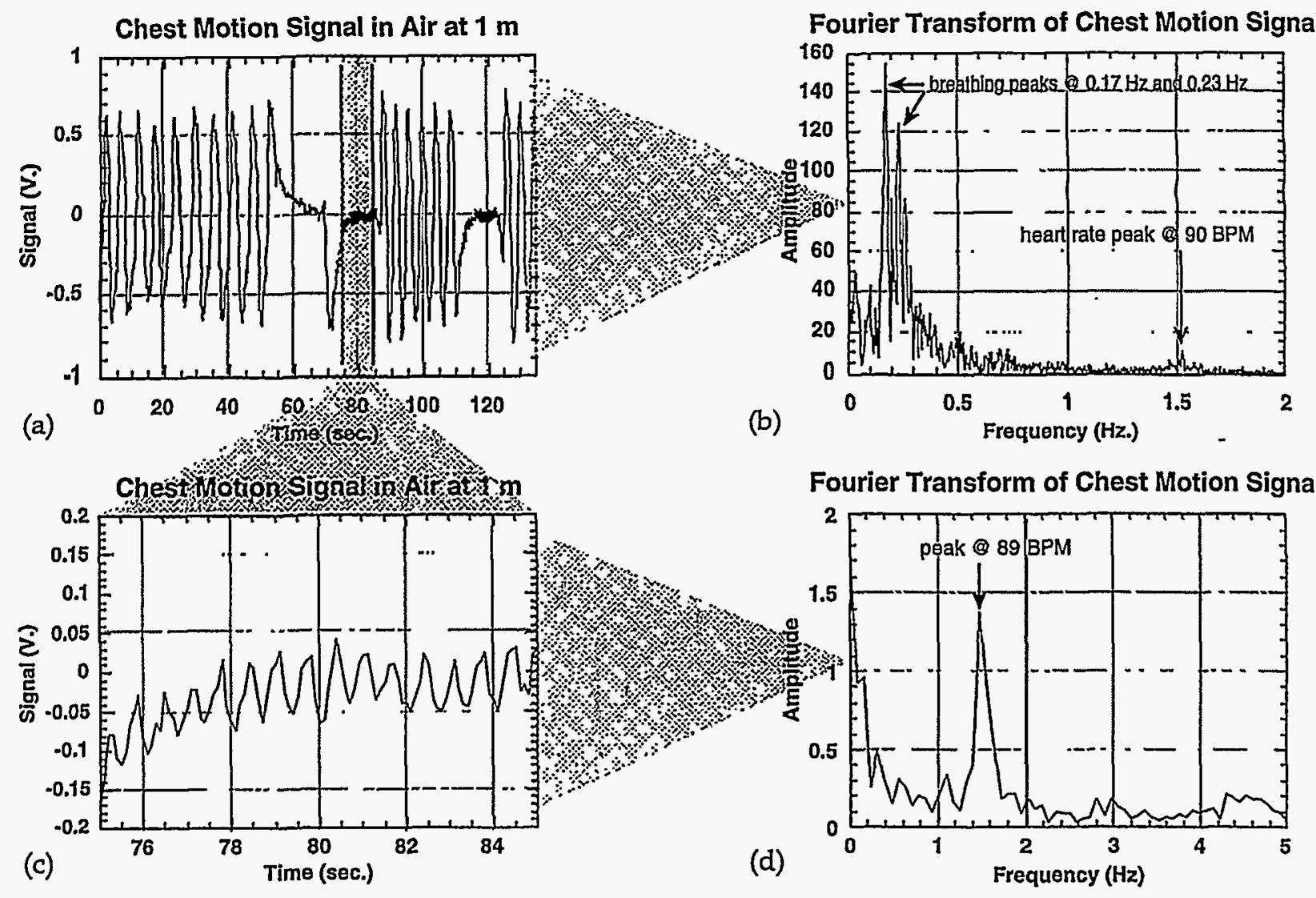

Figure 5. Breathing motion and heart beat signals recorded with a human subject sitting still in a chair by the RRR through air from a distance of 1 meter. (a) the raw RRR signal showing clear oscillations due to the breathing motion of the subject. At two points in time, the subject held his breath for short periods. These periods are seen as flat spots in the plot. Note that the breathing frequency is higher just after the breath holding periods. (b) the Fourier transform of the breathing signal showing two prominent peaks at the two dominant breathing frequencies as well as a small peak at the heart rate. (c) an expanded plot of the highlighted section in (a) showing the oscillations due to the heart beat during a breath holding period. (d) the Fourier transform of the heart beat signal in (c) displaying a peak at the heart rate of 89 beats per minute.

approximately one meter away from the $R R R$ with his chest directly aligned with the center of the RRR. The subject sat as still as possible and breathed normally. On several occasions during the data acquisition, the subject held his breath. The results of these tests are presented in Figure 5. The overall chest motion signal and its FT are presented in Figure 5a and b. Clearly visible are the peaks due to chest motion associated with breathing. Also visible in Figure $5 \mathrm{~b}$ is a peak corresponding to the heart beat. The presence of heart beat signal is further demonstrated in Figures $5 c$ and $d$, which focus on a segment of the signal corresponding to a period during which the subject held his breath. Figure $5 c$ is a blow-up of this segment and Figure $5 d$ is the FT of just this segment. The peak in the FT at the heart rate stands well above the background. This is a demonstration of the sensitivity of the $R R R$, and in particular, the gain and increased signal-to-noise afforded by the parabolic antenna design.

\section{Concept for the Next Generation RRR}

The results with the first generation prototype $R R R$ indicate great promise for the use of microwave motion sensors in urban search-andrescue (USAR). As discussed above, this first prototype RRR uses MIR technology which is inherently low power and limited in its range of application. The conditions of USAR naturally require high signal-to-noise ratio $(S / N)$ and high sensitivity while demanding a great deal of background noise rejection. The need for very 
Table 2.

Specifications for the Next Generation RRR (RRR2)

$\begin{array}{ll}\text { Center frequency } & 1 \mathrm{GHz} \\ \text { Output power } & \text { several Watts } \\ \text { Output profile } & 10 \mathrm{~ns} \text { bursts of } 1 \mathrm{GHz} \mathrm{RF} \\ \text { Pulse repetition frequency (PRF) } & 1-10 \mathrm{kHz} \\ \text { Power } & \text { rechargeable gel cells (as required) } \\ \text { Approximate dimensions } & 0.75 \mathrm{~m} \mathrm{w} \times 0.75 \mathrm{~m} \mathrm{~h} \times 0.5 \mathrm{~m} \mathrm{~d} \\ \text { Estimated weight (incl. batteries) } & 20 \mathrm{~kg} \\ \text { Target operation time (1 charge) } & 10 \mathrm{hr} .\end{array}$

Possible antennas:

1. Horn transmit antenna

Parabolic dish receive antenna

2. Single transmit/receive collimating horn with recirculator

small, lightweight and low power devices is, however, somewhat relaxed since it is common to bring large amounts of equipment and manpower to disaster sites. Based on what has been learned from the experiments described herein, we have begun to develop a concept for the next generation RRR device (hereafter referred to as RRR2).

In order to improve the $S / N$ and increase the operable depth of penetration through rubble, the next generation device must have a significantly higher output power than that achievable with current MIR technology. RRR2 would also have the capability to operate at a slightly lower center frequency for greater penetration through concrete. Because the types of structures and construction materials vary with geographical location, RRR2 may be designed to operate at two or more different, user selectable, center frequencies for optimal performance in different USAR conditions.

The RRR2 would transmit short bursts of RF at an average power of several watts. The burst length would be chosen to be long enough so that the spectrum of the output would be nearly monochromatic, thus allowing high performance horn antennas to be used. Narrow-band operation will allow the use of a well tuned receiver which will in turn significantly reduce the susceptibility to background noise. The use of short bursts has the added advantage of allowing the device to be "range-gated" which can be used to provide rough indication of the distance from which the signal is coming, as well as aiding in the rejection of background noise. The received signal could be "mixed" with a time correlated reference signal, to give very high sensitivity to small motions within the range gate.

The output of the RRR2's radar system would go to a portable computer or digital signal processing (DSP) device which would be carried with the operator. The DSP device would implement a specially-designed algorithm to analyze the signals on the fly, and trigger an alarm upon recognition of a live victim. More sophisticated spectral analysis methods beyond simple Fourier techniques are also planned for RRR2. Heavier components of the system, such as the batteries and the computer, would be carried in a backpack or belt-mount, leaving the operator's hands free to position the lightweight antenna. A list of some roughly estimated specifications of the RRR2 is presented in Table 2.

Our plan is to work with others in the USAR community to develop this next-generation $R R R$ device. The first generation system has been delivered and continues to undergo tests. The success and experience gained with the present device will provide valuable guidance for future developments. By partnering with government and industrial organizations that have an interest 
in remote detection of live personnel, we plan ongoing refinements to the RRR, and expect spinoff technologies through new research and development.

\section{Summary and Conclusions}

A first generation prototype microwave motion sensor device specifically designed to find live personnel trapped in collapsed structures has been designed and tested. The device, called the Rubble Rescue Radar (RRR) incorporates Micropower Impulse Radar (MIR) technology which was developed at Lawrence Livermore National Laboratory (LLNL) over the last 3 years. A special rubble pile testbed was also built on site at LLNL for use in the tests of the RRR. The testbed was designed to evaluate the RRR's performance under conditions similar to those found in USAR situations, and to allow tests to be conducted with human subjects.

The results with the first generation prototype $R R R$ indicate great promise for the use of microwave motion sensors in USAR. The tests conducted with the current RRR prototype also point out its limitations and help determine the requirements for a next generation device which would be practical for use in real-life USAR operations. As a result, a conceptual design for this next generation device (RRR2) has emerged, and some of its specifications have been estimated. We are currently seeking government and industrial partners for building and testing the RRR2, and to provide support that will allow us to extend our efforts in remote personnel detection.

\section{Acknowledgments}

The author would like to thank Michael A. Dillabough of the Army Corps of Engineers Earthquake Preparedness Center of Expertise in San Francisco for funding the initial phases of this work and technical information on search-andrescue techniques and problems. Gratitude goes to Stephen Azevedo, John Warhus, Brooks Johnston, Gregory Burnett, Forrest Lewis and John McWilliams of Lawrence Livermore National Laboratory for help on various aspects of the RRR project. The author also thanks Vince Bormolini of Western Concrete for providing the concrete. pipes, and for information on the ASTM codes.

\section{References}

1. S. G. Azevedo and T. E. McEwan, "Micropower Impulse Radar", Science and Technology Review, January/February 1996, pp. 16-29.

2. W. S. Haddad, E. T. Rosenbury, K. B. Johnson and F. J. Pearce, "Measuerments of the Dielectric Properties of Body Tissues and Fluids at Microwave Frequencies", manuscript in preparation.

3. S. G. Azevedo and T. E. McEwan, "Micropower Impulse Radar", Science and Technology Review, January/February 1996, pp. 28-29.

4. J. F. Holzrichter, W. A. Lea, T. E. McEwan, L. C. Ng, G. C. Burnett (1996), "Speech Coding, Recognition, and Synthesis using Radar and Acoustic Sensors", University of California Report UCRL-ID-123687, (reprints available from the Office of Scientific and Technical Information, P.O.Box 62, Oak Ridge, TN, 37831) 